\title{
Terpyridine Molybdenum Dinitrogen Chemistry: Synthesis of Dinitrogen Complexes that Vary by Five Oxidation States
}

\author{
Máté J. Bezdek, Sheng Guo and Paul J. Chirik* \\ Department of Chemistry, Princeton University, Princeton, New Jersey 08540 \\ pchirik@princeton.edu
}

\section{Table of Contents}

$\begin{array}{ll}\text { Additional NMR Spectroscopic Data } & \text { S2 }\end{array}$

Additional Raman Spectroscopic Data $\quad$ S4

$\begin{array}{ll}\text { DFT Input File Examples } & \text { S7 }\end{array}$

Coordinates from the Geometry Optimizations $\quad$ S13

DFT-Computed Qualitative Molecular Orbital Diagram for $\quad$ S19

$\left[\left({ }^{\mathrm{Ph}} \mathrm{Tpy}\right)\left(\mathrm{PPh}_{2} \mathrm{Me}\right)_{2} \mathrm{Mo}(\mathrm{Cl})\right]$

$\begin{array}{ll}\text { Additional Mulliken Spin Density Plots } & \text { S20 }\end{array}$ 


\section{Additional NMR Spectroscopic Data}

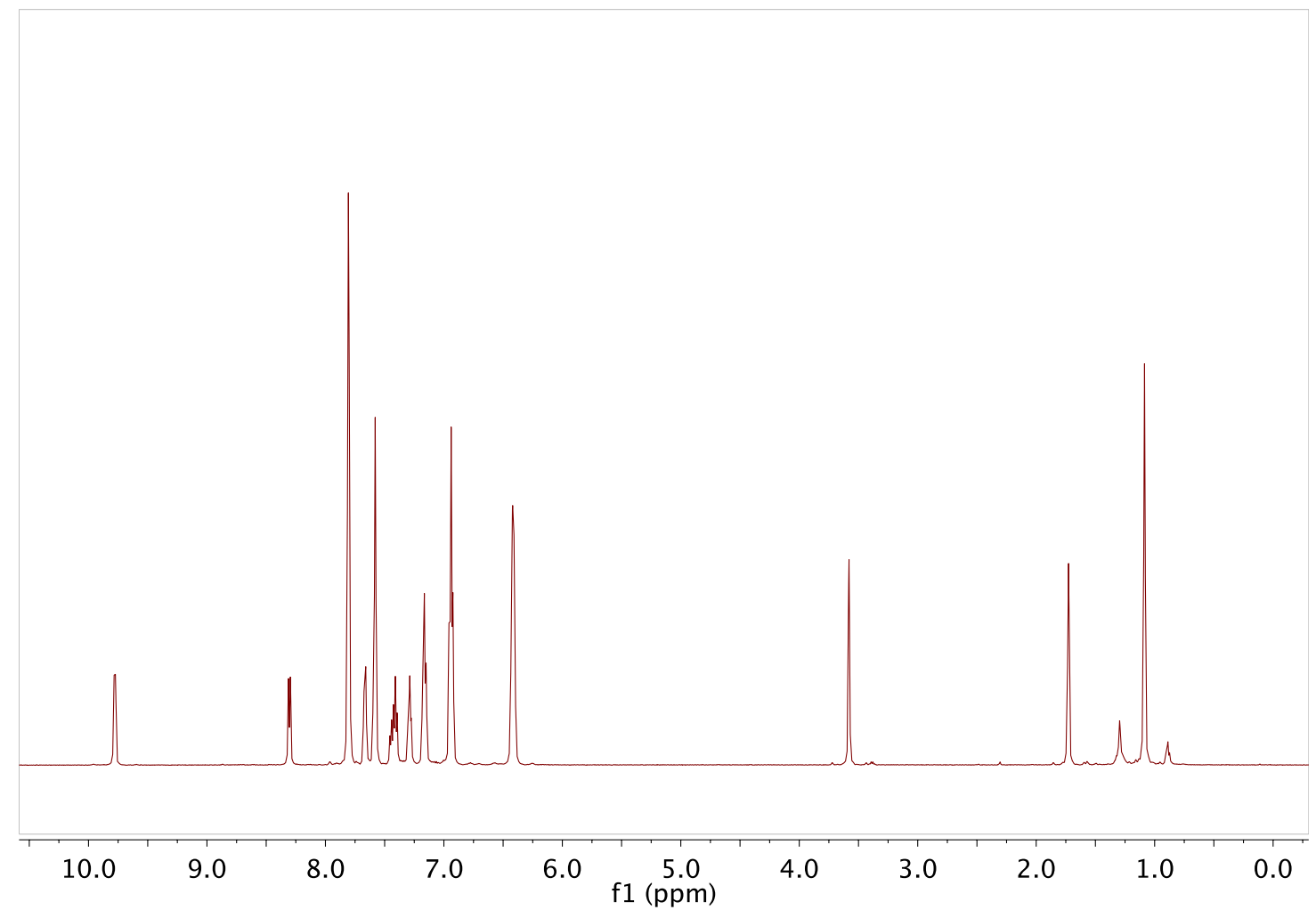

Figure S1. THF- $d_{8}{ }^{1} \mathrm{H}$ NMR spectrum of $\left[\left\{\left({ }^{\mathrm{Ph}} \mathrm{Tpy}\right)\left(\mathrm{PPh}_{2} \mathrm{Me}\right)_{2} \mathrm{Mo}\right\}_{2}\left(\mu_{2}-\mathrm{N}_{2}\right)\right]\left[\mathrm{BArF}{ }^{24}\right]_{2}([\mathbf{1}-$ $\left.\mathbf{N}_{2}\right]^{2+}$ ) at $295 \mathrm{~K}$.

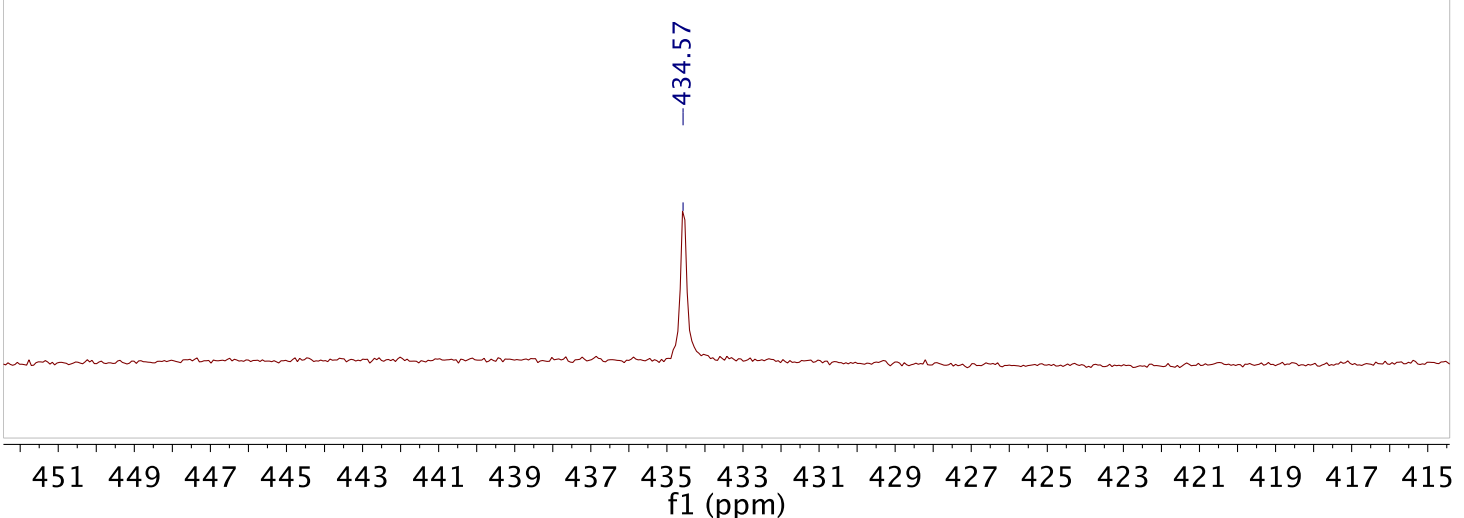

Figure S2. THF- $d_{8}{ }^{15} \mathrm{~N}$ NMR spectrum of $\left[\left\{\left({ }^{\mathrm{Ph}} \mathrm{Tpy}\right)\left(\mathrm{PPh}_{2} \mathrm{Me}\right)_{2} \mathrm{Mo}\right\}_{2}\left(\mu_{2}-{ }^{15} \mathrm{~N}_{2}\right)\right]\left[\mathrm{BArF}{ }^{24}\right]_{2}$ $\left(\left[1-{ }^{15} \mathbf{N}_{2}\right]^{2+}\right)$ at $295 \mathrm{~K}$. 


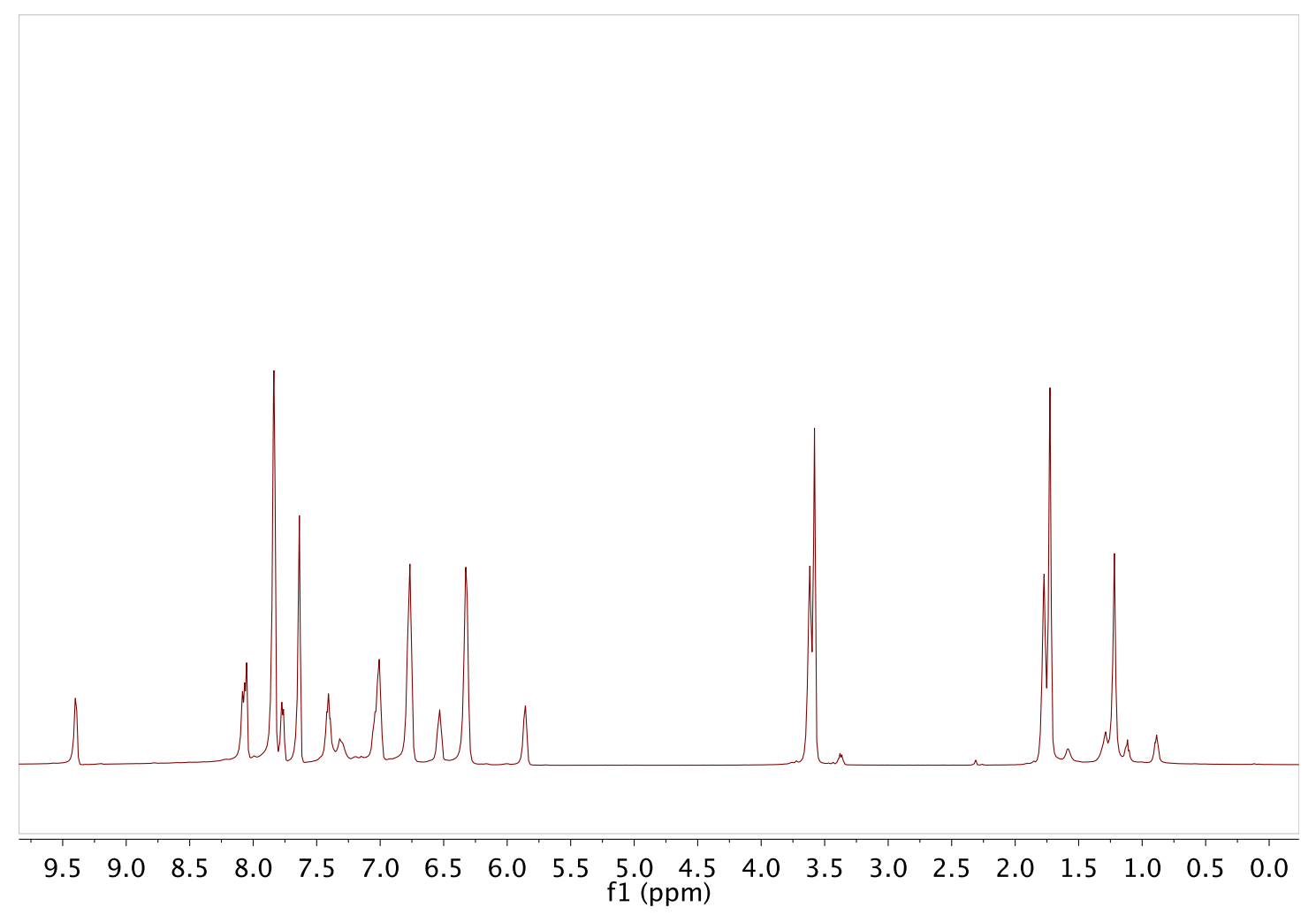

Figure S3. THF- $d_{8}{ }^{1} \mathrm{H}$ NMR spectrum of $\left[\left({ }^{\mathrm{Ph}} \mathrm{Tpy}\right)\left(\mathrm{PPh}_{2} \mathrm{Me}\right)_{2} \mathrm{Mo}\right]_{2}\left(\mu_{2}-\mathrm{N}_{2}\right)\left(\left[\mathbf{1}-\mathbf{N}_{\mathbf{2}}\right]\right)$ at 295 K.

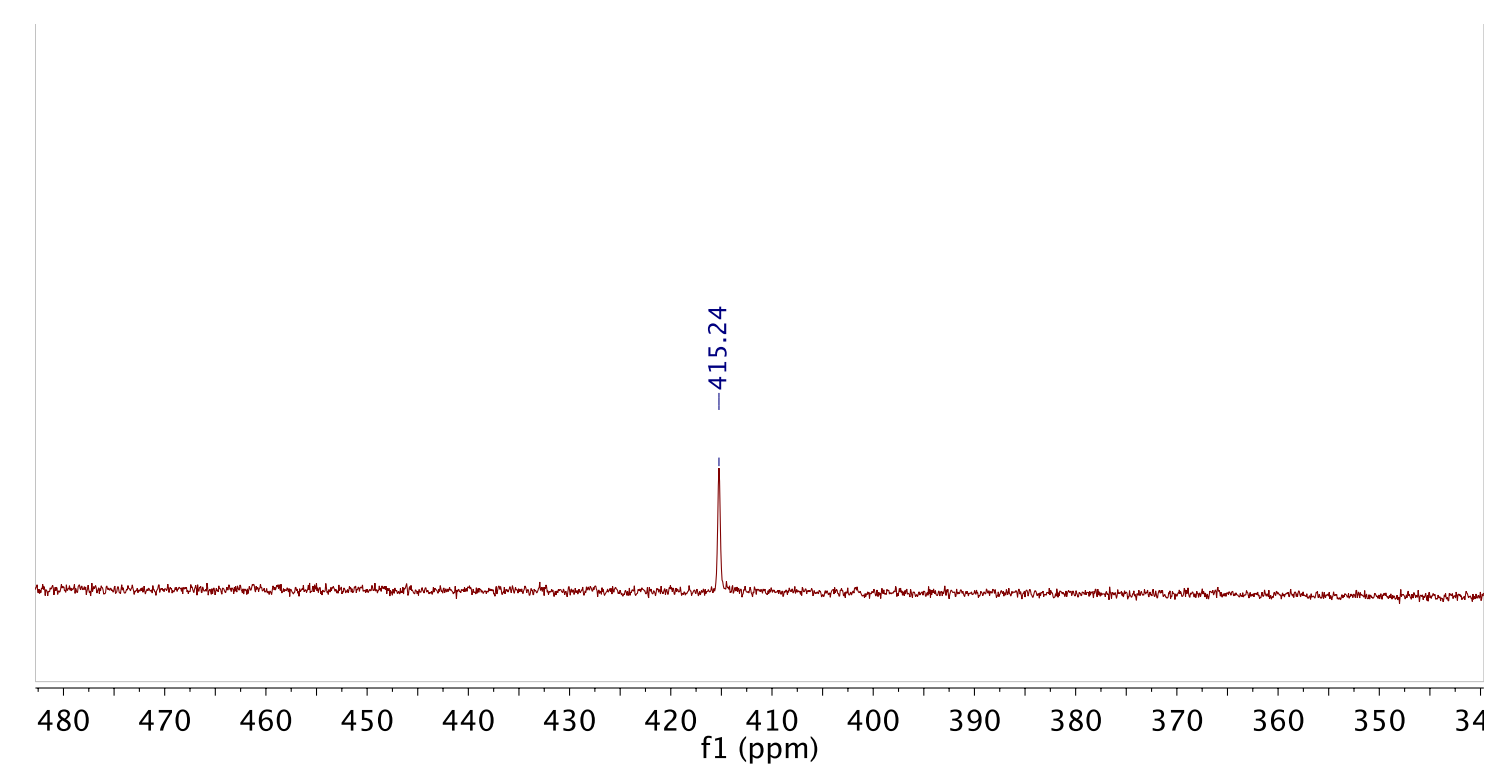

Figure S4. THF- $d_{8}{ }^{15} \mathrm{~N}$ NMR spectrum of $\left[\left({ }^{\mathrm{Ph}} \mathrm{Tpy}\right)\left(\mathrm{PPh}_{2} \mathrm{Me}\right)_{2} \mathrm{Mo}\right]_{2}\left(\mu_{2^{-}}{ }^{15} \mathrm{~N}_{2}\right)\left(\left[\mathbf{1 -}^{15} \mathrm{~N}_{2}\right]\right)$ at 295 K. 


\section{Additional Raman Spectroscopic Data}

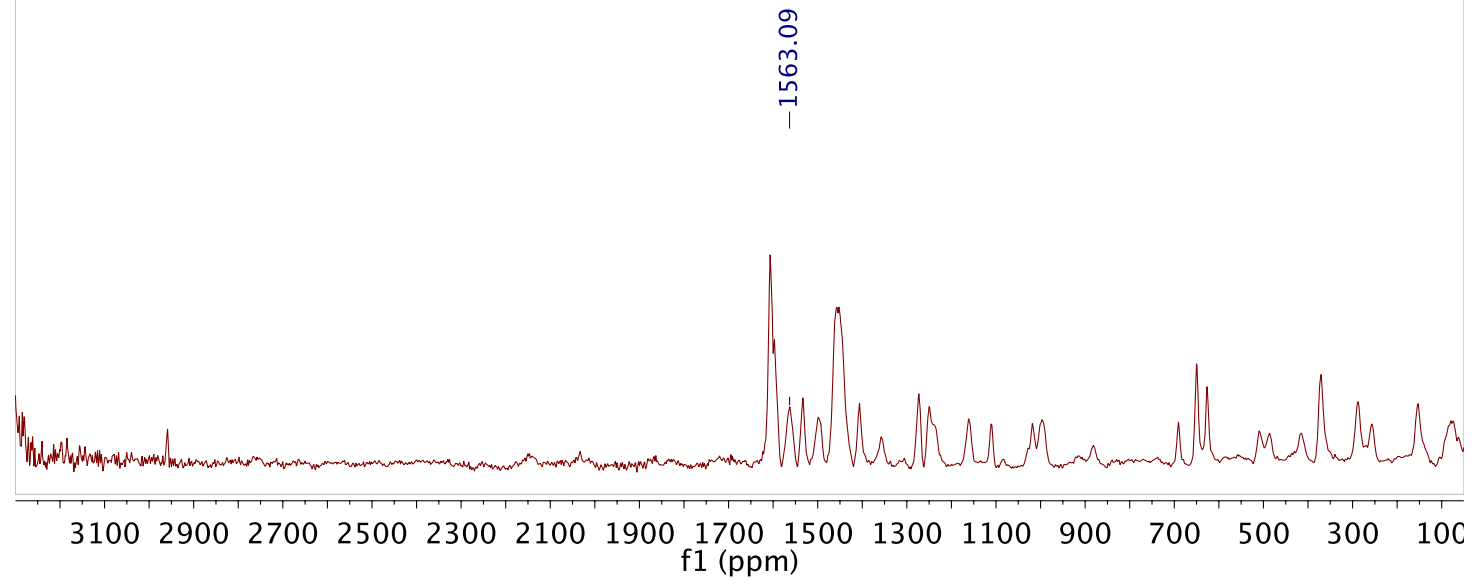

Figure S5. Raman spectrum of $\left[\left\{\left({ }^{\mathrm{Ph}} \mathrm{Tpy}\right)\left(\mathrm{PPh}_{2} \mathrm{Me}\right)_{2} \mathrm{Mo}\right\}_{2}\left(\mu_{2}-\mathrm{N}_{2}\right)\right]\left[\mathrm{BArF}{ }^{24}\right]_{2}\left(\left[\mathbf{1}-\mathbf{N}_{\mathbf{2}}\right]^{2+}\right)$ at $295 \mathrm{~K}$ in 2-Me-THF.

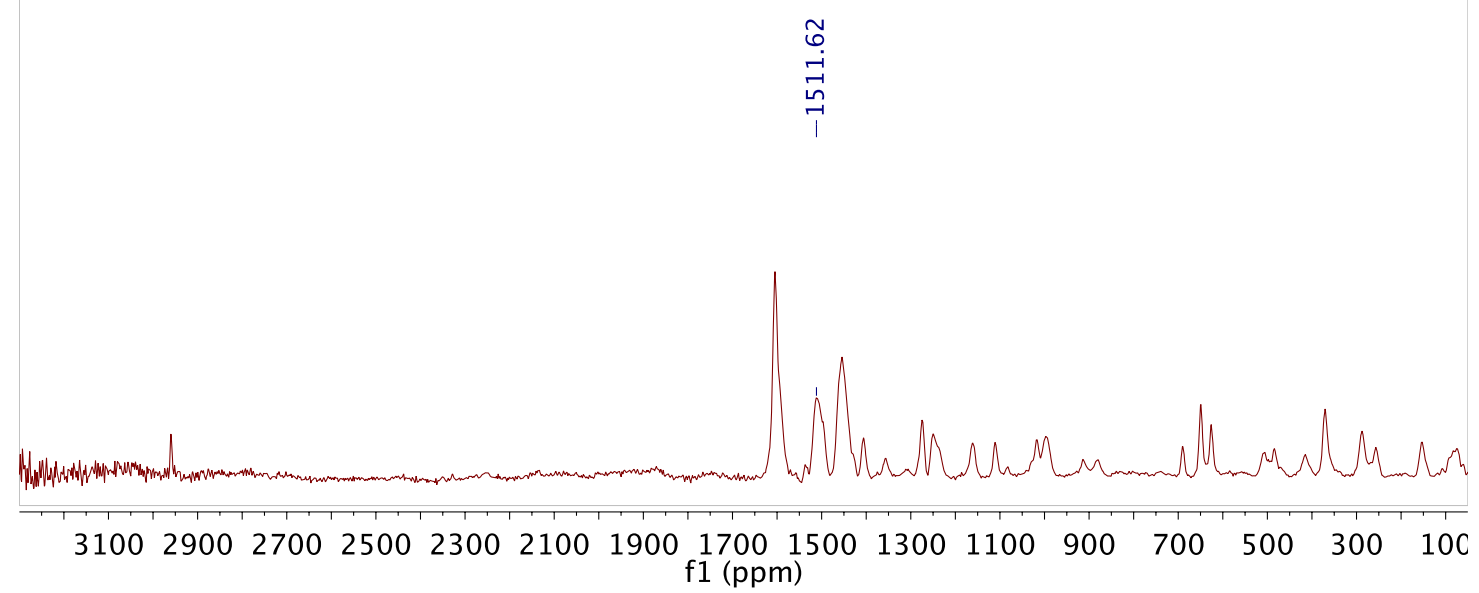

Figure S6. Raman spectrum of $\left[\left\{\left({ }^{\mathrm{Ph}} \mathrm{Tpy}\right)\left(\mathrm{PPh}_{2} \mathrm{Me}\right)_{2} \mathrm{Mo}\right\}_{2}\left(\mu_{2^{-}}{ }^{15} \mathrm{~N}_{2}\right)\right]\left[\mathrm{BArF}{ }^{24}\right]_{2}\left(\left[1-{ }^{15} \mathbf{N}_{2}\right]^{2+}\right)$ at $295 \mathrm{~K}$ in 2-Me-THF. 


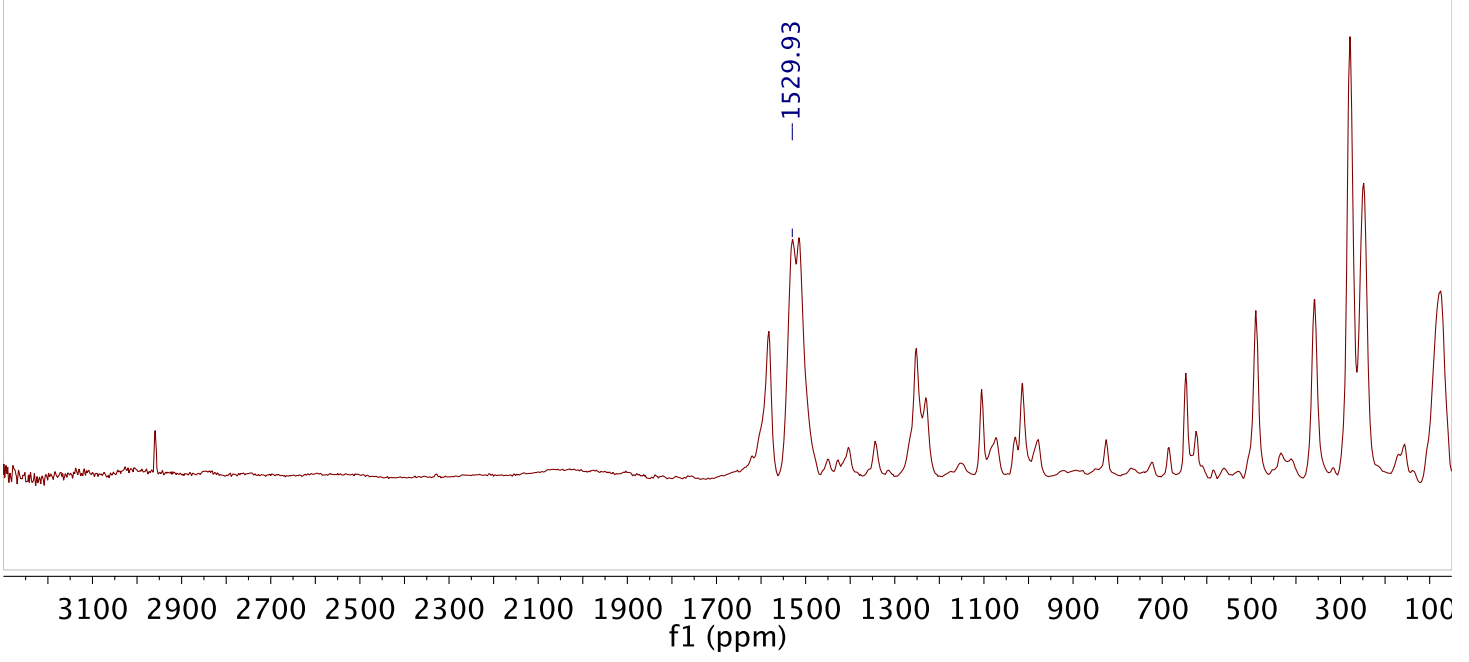

Figure S7. Raman spectrum of $\left[\left\{\left({ }^{\mathrm{Ph}} \mathrm{Tpy}\right)\left(\mathrm{PPh}_{2} \mathrm{Me}\right)_{2} \mathrm{Mo}\right\}_{2}\left(\mu_{2}-\mathrm{N}_{2}\right)\right]\left[\mathrm{BArF}{ }^{24}\right]\left(\left[\mathbf{1}-\mathbf{N}_{2}\right]^{+}\right)$at 295 $\mathrm{K}$ in 2-Me-THF.

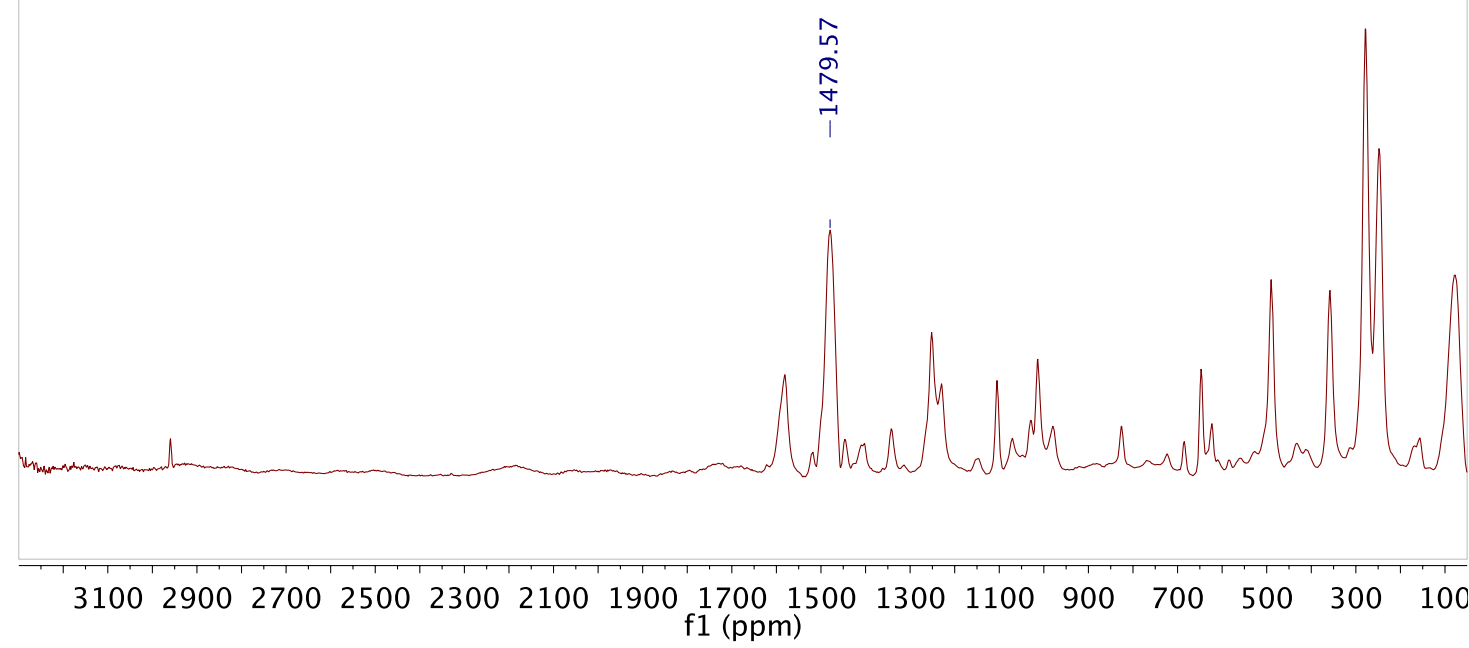

Figure S8. Raman spectrum of $\left[\left\{\left({ }^{\mathrm{Ph}} \mathrm{Tpy}\right)\left(\mathrm{PPh}_{2} \mathrm{Me}\right)_{2} \mathrm{Mo}\right\}_{2}\left(\mu_{2^{-}}{ }^{15} \mathrm{~N}_{2}\right)\right]\left[\mathrm{BArF}^{24}\right]\left(\left[\mathbf{1}^{-15} \mathbf{N}_{\mathbf{2}}\right]^{+}\right)$ at $295 \mathrm{~K}$ in $2-\mathrm{Me}-\mathrm{THF}$. 


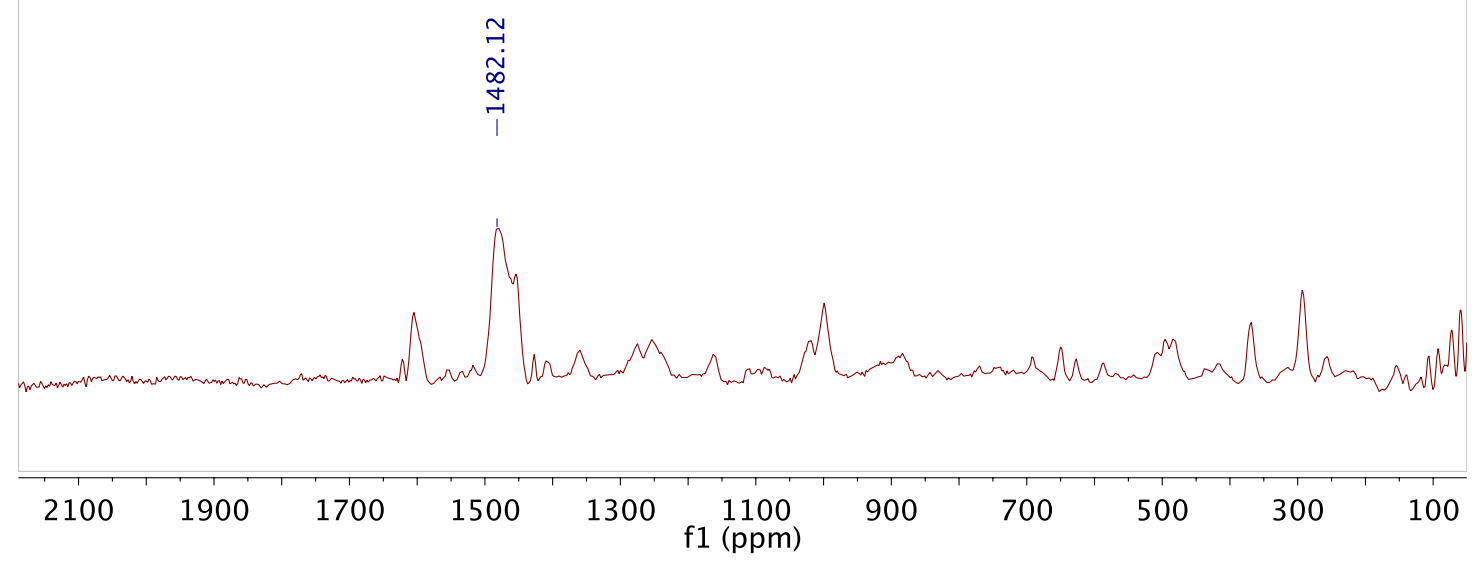

Figure S9. Raman spectrum of $\left[\left\{\left({ }^{\mathrm{Ph}} \mathrm{Tpy}\right)\left(\mathrm{PPh}_{2} \mathrm{Me}\right)_{2} \mathrm{Mo}\right\}_{2}\left(\mu_{2}-\mathrm{N}_{2}\right)\right]\left[\mathrm{BArF}{ }^{24}\right]_{3}\left(\left[\mathbf{1}-\mathbf{N}_{\mathbf{2}}\right]^{3+}\right)$ at $295 \mathrm{~K}$ in 2-Me-THF.

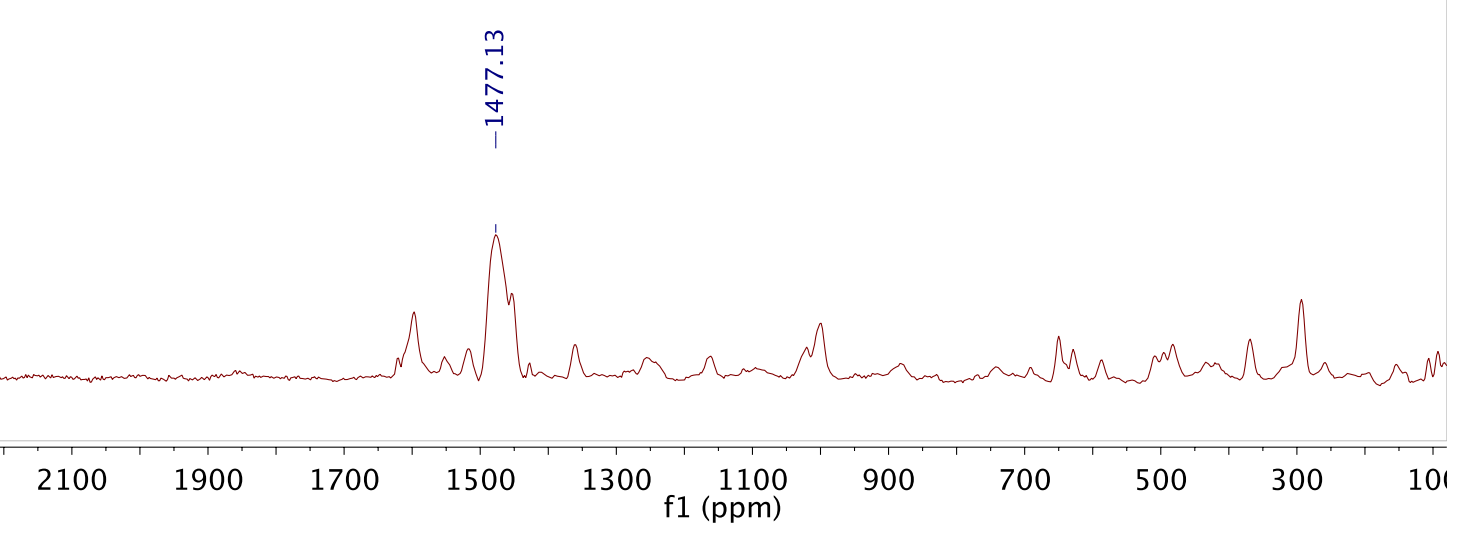

Figure S10. Raman spectrum of $\left[\left\{\left({ }^{\mathrm{Ph}} \mathrm{Tpy}\right)\left(\mathrm{PPh}_{2} \mathrm{Me}\right)_{2} \mathrm{Mo}_{2}\left(\mu_{2}-\mathrm{N}_{2}\right)\right]\left[\mathrm{BArF}^{24}\right]_{4}\left(\left[\mathbf{1 - N _ { 2 }}\right]^{4+}\right)\right.$ at $295 \mathrm{~K}$ in 2-Me-THF.

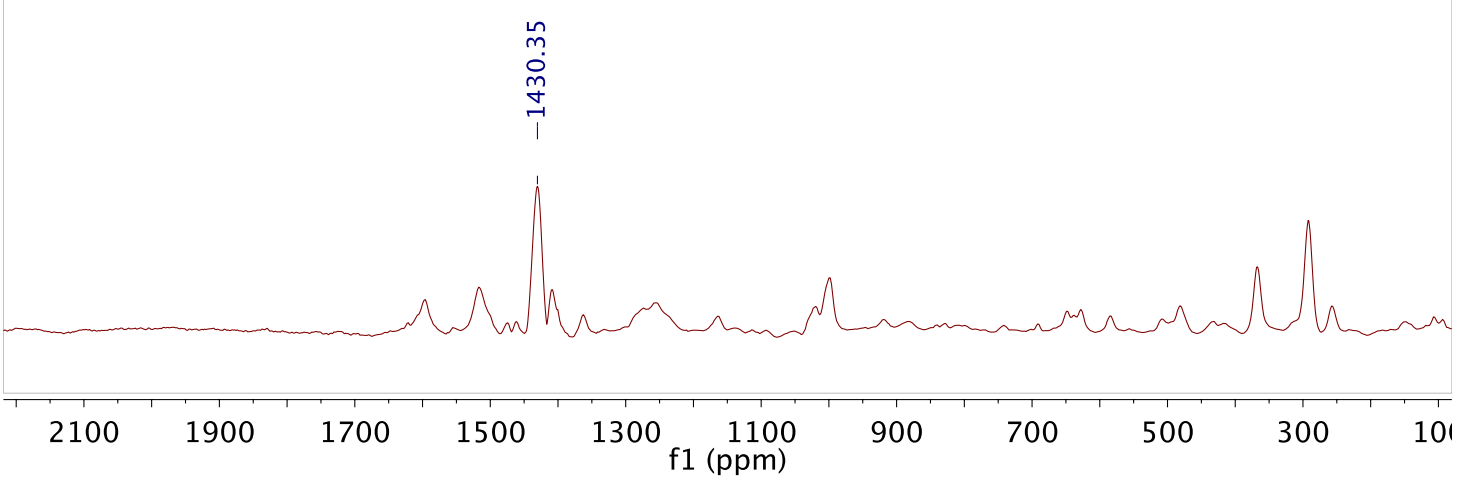

Figure S11. Raman spectrum of $\left[\left\{\left({ }^{\mathrm{Ph}} \mathrm{Tpy}\right)\left(\mathrm{PPh}_{2} \mathrm{Me}\right)_{2} \mathrm{Mo}\right\}_{2}\left(\mu_{2^{-}}{ }^{15} \mathrm{~N}_{2}\right)\right]\left[\mathrm{BArF}^{24}\right]_{4}\left(\left[\mathbf{1 -}^{15} \mathbf{N}_{2}\right]^{4+}\right)$ at $295 \mathrm{~K}$ in $2-\mathrm{Me}-\mathrm{THF}$. 


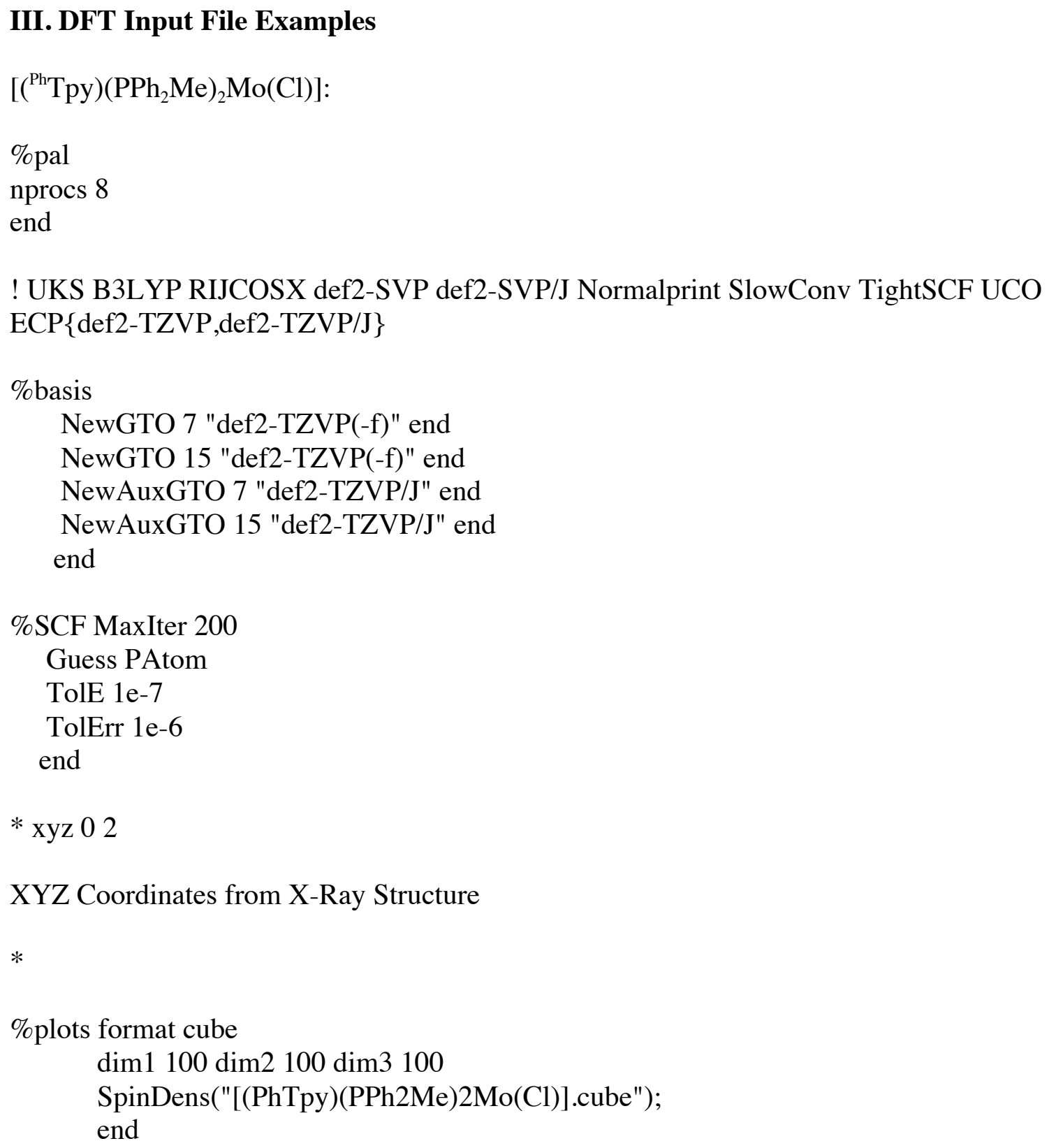


$\left.\left[\left\{{ }^{\mathrm{Ph}} \mathrm{Tpy}\right)\left(\mathrm{PPh}_{2} \mathrm{Me}\right)_{2} \mathrm{Mo}\right\}_{2}\left(\mu_{2}-\mathrm{N}_{2}\right)\right]\left[\mathrm{BArF}^{24}\right]_{2}\left(\left[\mathbf{1}-\mathbf{N}_{\mathbf{2}}\right]^{2+}\right)$ :

! RKS B3LYP RIJCOSX def2-SVP def2-SVP/J Normalprint SlowConv TightSCF Opt Pal8 UCO

\%basis NewGTO 42 "def2-TZVP(-f)" end NewGTO 7 "def2-TZVP(-f)" end

NewGTO 15 "def2-TZVP(-f)" end

NewAuxGTO 42 "def2-TZVP/J" end

NewAuxGTO 7 "def2-TZVP/J" end

NewAuxGTO 15 "def2-TZVP/J" end

end

\%SCF MaxIter 500

TolE 1e-7

TolErr 1e-6

end

*xyz 21

XYZ Coordinates of the dicationic fragment of the molecule from X-Ray structure 


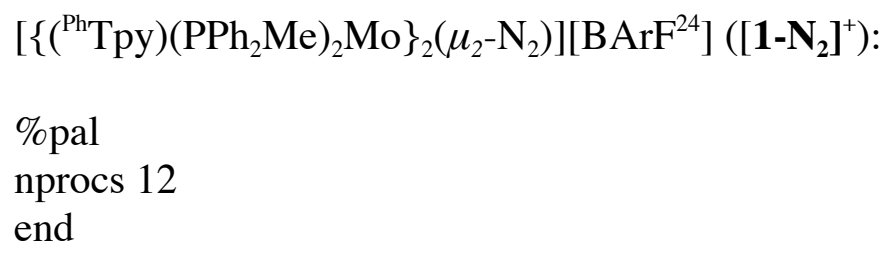




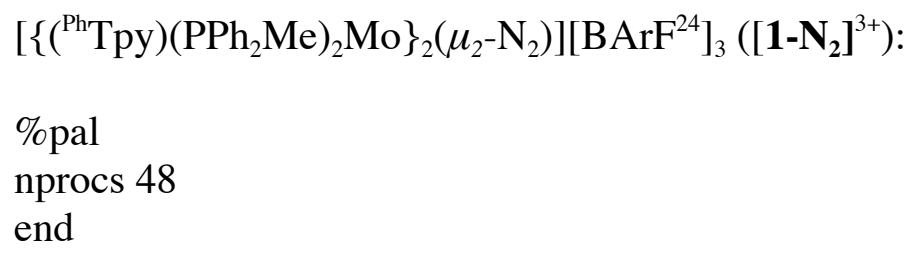




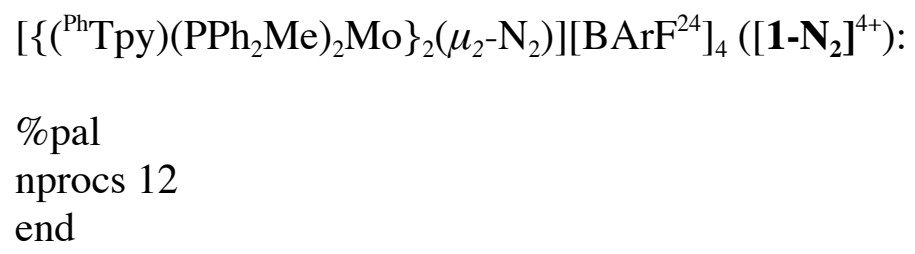


$\left[{ }^{\mathrm{Ph}} \mathrm{Tpy}\right)\left(\mathrm{PPh}_{2} \mathrm{Me}\right)_{2} \mathrm{Mo}_{2}\left(\mu_{2}-\mathrm{N}_{2}\right)\left(\left[\mathbf{1}-\mathbf{N}_{2}\right]\right):$

$\%$ pal

nprocs 12

end

! RKS B3LYP RIJCOSX def2-SVP def2-SVP/J Normalprint SlowConv TightSCF UCO ECP $\{$ def2-TZVP,def2-TZVP/J\}

\%basis

NewGTO 7 "def2-TZVP(-f)" end

NewGTO 15 "def2-TZVP(-f)" end

NewAuxGTO 7 "def2-TZVP/J" end

NewAuxGTO 15 "def2-TZVP/J" end

end

\%SCF MaxIter 200

TolE 1e-7

TolErr 1e-6

end

*xyz 01

Geometry-Optimized XYZ Coordinates of $\left(\left[\mathbf{1}-\mathbf{N}_{2}\right]^{2+}\right)$

* 


\section{Coordinates from Geometry Optimizations}

$\left[\left({ }^{\mathrm{Ph}} \mathrm{Tpy}\right)\left(\mathrm{PPh}_{2} \mathrm{Me}\right)_{2} \mathrm{Mo}(\mathrm{Cl})\right]$

\begin{tabular}{|c|c|c|c|}
\hline & 39 & 1058433608 & \\
\hline & 8848807241602 & -4.47306745916254 & 2047 \\
\hline & .26290234966920 & -4.73618261672074 & -1.67068738 \\
\hline & 25936678510821 & -3.65946083079741 & 326 \\
\hline & 18173472829932 & -2.34198228202380 & -2.05600 \\
\hline & 8526811708 & 121 & -2.8910 \\
\hline & .20030853313865 & -1.13338134926011 & -4.28249950 \\
\hline & 0.13199203497770 & 0.07578 & -5.00142 \\
\hline & 0.0244471 & 06939450 & -4.2 \\
\hline $\mathrm{C}$ & -0.0001518 & 6193 & -2.8 \\
\hline & 596 & & -1.9 \\
\hline 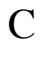 & 835 & 7704 & -2.4 \\
\hline & 139 & & \\
\hline 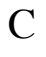 & 870 & 431 & -0 \\
\hline C & & & \\
\hline & 0.1 & 469 & 229 \\
\hline C & & & \\
\hline & 0.9 & -0.8 & -8.6 \\
\hline C & & 0.1 & -9.31 \\
\hline C & -0. & & -8. \\
\hline 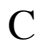 & & & \\
\hline 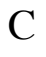 & & -1.2 & \\
\hline C & 3.4 & -0.4 & -1.27 \\
\hline 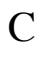 & 4.2 & -1. & -1.3 \\
\hline C & 4.90 & -1.9 & -2.5 \\
\hline C & & -1.1 & -3.6 \\
\hline C & 4.0 & -0.0( & -3.61 \\
\hline C & 3.3 & 0.3 & 288 \\
\hline$r$ & 3.3 & & 453 \\
\hline C & 4.5 & 2.0 & 381 \\
\hline & 5.13 & $3.1^{\prime}$ & 572 \\
\hline $\mathrm{C}$ & 4.5 & 3.7 & 996 \\
\hline $\mathrm{C}$ & 2291360190 & $3.21704061 \mathrm{~S}$ & 3443 \\
\hline $\mathrm{C}$ & & & \\
\hline $\mathrm{C}$ & 51987040603 & 3607 & 63241 \\
\hline C & -3.3 & & 4419 \\
\hline 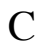 & 52046120076 & 99097 & 80605 \\
\hline 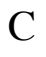 & 5234915668 & -0.10 & 71268 \\
\hline $\mathrm{C}$ & -4.52967548689784 & 1.10365442229570 & 40037 \\
\hline 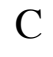 & -4.65306155743324 & & -2.90 \\
\hline $\mathrm{C}$ & -4.09053646752996 & 1.63778871870744 & -1.66656811634671 \\
\hline & -3.41380269448521 & -1.51028935912752 & 0.66945583844022 \\
\hline
\end{tabular}




\begin{tabular}{|c|c|c|c|}
\hline & -2.9090038098 & 3452395484901 & \\
\hline & -3.54445790162240 & -3.25614379323447 & 920 \\
\hline & -4.687979973 & 99043 & 460 \\
\hline & 723 & & \\
\hline & -4.566664607 & 42580 & 89 \\
\hline & & -2.0 & \\
\hline & 0.06226610 & 0.022 & -2.1 \\
\hline & 62343 & & 033 \\
\hline & 2.54141290229546 & -0.03 & 0.22 \\
\hline & 27345 & -0.0 & 4692 \\
\hline & 426181043 & 823132 & 5760 \\
\hline & -0.0 & -0.0 & 401 \\
\hline & 0.05 & -2.9 & \\
\hline & 0.1 & -5.2 & 0.4 \\
\hline & 0.3 & -5.7 & \\
\hline & 0.31 & -3.8 & -3.6 \\
\hline I & 0.2 & -2.0 & \\
\hline & -0.0 & & 408 \\
\hline $\mathrm{H}$ & -0.1 & & \\
\hline & -0.2 & & 802 \\
\hline $\mathrm{I}$ & -0.1 & & \\
\hline & -0.0 & & 87 \\
\hline & 1.49 & -1.6 & -6.675 \\
\hline & 1.5 & -1.5 & -9 \\
\hline & & & \\
\hline & -1.0 & & 041 \\
\hline I & 94404 & & 416 \\
\hline I & 2.75 & -2.2 & \\
\hline & 4.14 & -1.2 & \\
\hline $\mathrm{I}$ & 2.5 & $-1 .($ & 2.3 \\
\hline & 4.38 & -2.3 & -0.50470 \\
\hline $\mathrm{H}$ & 5.5 & -2.9 & -2.6 \\
\hline & 5.28 & -1.4 & -4 \\
\hline 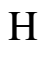 & 3.90 & 0.6 & -4. \\
\hline & 2.78 & 1.2 & -2.38196 \\
\hline $\mathrm{H}$ & 4.99 & 1.5 & -0.5 \\
\hline & 6.03 & & \\
\hline H & 5.0 & & \\
\hline & 03901 & 3.6 & 3.472492 \\
\hline H & 1.94 & 1.66 & 2.480376 \\
\hline & 8924428158 & & 192135 \\
\hline & -2.76 & & \\
\hline & 860701983 & 1.243 & 1.36894862682325 \\
\hline & -2.71 & -1.3 & -2.50189407718226 \\
\hline & -3.72524917682672 & -0.78679286945769 & -4.68158376083342 \\
\hline $\mathbf{H}$ & -4.9718835 & 1.367742519851 & -4 \\
\hline
\end{tabular}




$\begin{array}{cccr}H & -5.19277423240416 & 2.91586296529713 & -3.01550624656293 \\ H & -4.20378137314519 & 2.33322527291514 & -0.83317954353635 \\ H & -2.02109106357319 & -1.72594989355332 & 2.31628521569815 \\ H & -3.13782266778976 & -3.72460870113552 & 3.26603754275102 \\ H & -5.18435810920809 & -4.65905359333995 & 2.17709535786943 \\ \text { H } & -6.08874652427617 & -3.57379706291087 & 0.11955391108213 \\ \text { H } & -4.98750305938031 & -1.57455986050795 & -0.82486101011943\end{array}$

$$
\left[\left\{\left({ }^{\mathrm{Ph}} \mathrm{Tpy}\right)\left(\mathrm{PPh}_{2} \mathrm{Me}\right)_{2} \mathrm{Mo}\right\}_{2}\left(\mu_{2}-\mathrm{N}_{2}\right)\right]\left[\mathrm{BArF}^{24}\right]_{2}\left(\left[\mathbf{1}-\mathbf{N}_{2}\right]^{2+}\right)
$$

$\begin{array}{lrrr}\mathrm{C} & 1.458573 & 2.311906 & 5.610739 \\ \mathrm{C} & 2.094244 & 3.314212 & 6.328706 \\ \mathrm{C} & 2.094503 & 3.250399 & 7.729556 \\ \mathrm{C} & 1.461611 & 2.170806 & 8.336465 \\ \mathrm{C} & 0.833063 & 1.186636 & 7.558424 \\ \mathrm{C} & 0.159314 & 0.008999 & 8.114466 \\ \mathrm{C} & 0.041899 & -0.282907 & 9.477148 \\ \mathrm{C} & -0.624631 & -1.441844 & 9.878362 \\ \mathrm{C} & -1.163549 & -2.283935 & 8.907530 \\ \mathrm{C} & -1.019870 & -1.953822 & 7.556074 \\ \mathrm{C} & -1.565696 & -2.723324 & 6.439680 \\ \mathrm{C} & -2.352863 & -3.875552 & 6.588596 \\ \mathrm{C} & -2.865986 & -4.521513 & 5.470436 \\ \mathrm{C} & -2.575081 & -3.986961 & 4.206705 \\ \mathrm{C} & -1.792855 & -2.845837 & 4.117305 \\ \mathrm{C} & -2.784651 & 2.423121 & 4.379824 \\ \mathrm{C} & -4.092603 & 2.931039 & 4.259181 \\ \mathrm{C} & -4.332115 & 4.133249 & 3.590527 \\ \mathrm{C} & -3.264948 & 4.864864 & 3.057135 \\ \mathrm{C} & -1.959830 & 4.386331 & 3.197314 \\ \mathrm{C} & -1.723032 & 3.166501 & 3.841356 \\ \mathrm{C} & -2.867620 & 1.398807 & 7.007917 \\ \mathrm{C} & -2.273485 & 2.563875 & 7.529730 \\ \mathrm{C} & -2.596188 & 3.020095 & 8.809711 \\ \mathrm{C} & -3.514955 & 2.317121 & 9.596912 \\ \mathrm{C} & -4.099241 & 1.150799 & 9.097197 \\ \mathrm{C} & -3.775520 & 0.691192 & 7.815759 \\ \mathrm{C} & -3.871107 & -0.201101 & 4.853082 \\ \mathrm{C} & 2.785216 & -1.872006 & 6.861914 \\ \mathrm{C} & 3.664496 & -1.016484 & 7.548635 \\ \mathrm{C} & 4.120342 & -1.340337 & 8.832036 \\ \mathrm{C} & 3.697495 & -2.517072 & 9.454556 \\ \mathrm{C} & 2.807021 & -3.366828 & 8.788644 \\ \mathrm{C} & 2.356265 & -3.047289 & 7.506639 \\ \mathrm{C} & 2.605625 & -3.110328 & 4.337066 \\ \mathrm{C} & 1.612580 & -4.095273 & 4.198680\end{array}$




\begin{tabular}{|c|c|c|c|}
\hline $\mathrm{C}$ & 1.932252 & -5.374290 & 3.726969 \\
\hline $\mathrm{C}$ & 3.245173 & -5.683235 & 3.361620 \\
\hline$C$ & 4.239125 & -4.706021 & 3.473987 \\
\hline $\mathrm{C}$ & 3.924715 & -3.435514 & 3.964140 \\
\hline $\mathrm{C}$ & 3.500013 & -0.382246 & 4.521420 \\
\hline $\mathrm{C}$ & 0.177355 & -3.081529 & 0.505002 \\
\hline $\mathrm{C}$ & 0.268434 & -4.433808 & 0.212698 \\
\hline $\mathrm{C}$ & 0.256823 & -4.849733 & -1.126293 \\
\hline $\mathrm{C}$ & 0.173432 & -3.873705 & -2.111426 \\
\hline $\mathrm{C}$ & 0.095648 & -2.517551 & -1.759094 \\
\hline $\mathrm{C}$ & 0.060835 & -1.423058 & -2.726043 \\
\hline $\mathrm{C}$ & 0.078934 & -1.577775 & -4.115990 \\
\hline $\mathrm{C}$ & 0.094405 & -0.447589 & -4.930906 \\
\hline $\mathrm{C}$ & 0.094261 & 0.814383 & -4.337337 \\
\hline $\mathrm{C}$ & 0.066420 & 0.920677 & -2.943886 \\
\hline $\mathrm{C}$ & 0.036465 & 2.181181 & -2.201317 \\
\hline $\mathrm{C}$ & 0.026052 & 3.441490 & -2.815643 \\
\hline $\mathrm{C}$ & -0.044608 & 4.596099 & -2.046273 \\
\hline $\mathrm{C}$ & -0.118956 & 4.457008 & -0.653710 \\
\hline $\mathrm{C}$ & -0.099498 & 3.184290 & -0.102136 \\
\hline $\mathrm{C}$ & -3.657319 & -1.207334 & 0.499089 \\
\hline $\mathrm{C}$ & -3.292616 & -2.538863 & 0.225515 \\
\hline $\mathrm{C}$ & -4.103821 & -3.607321 & 0.620776 \\
\hline $\mathrm{C}$ & -5.288860 & -3.365783 & 1.322778 \\
\hline $\mathrm{C}$ & -5.659151 & -2.049083 & 1.612508 \\
\hline $\mathrm{C}$ & -4.857869 & -0.980282 & 1.198217 \\
\hline $\mathrm{C}$ & -3.288671 & 0.441067 & -1.813853 \\
\hline $\mathrm{C}$ & -3.502538 & -0.644147 & -2.684163 \\
\hline $\mathrm{C}$ & -4.005557 & -0.442254 & -3.971323 \\
\hline $\mathrm{C}$ & -4.305499 & 0.848932 & -4.417956 \\
\hline $\mathrm{C}$ & -4.101845 & 1.935154 & -3.564892 \\
\hline $\mathrm{C}$ & -3.597698 & 1.734666 & -2.274952 \\
\hline $\mathrm{C}$ & -3.213664 & 1.634180 & 0.779506 \\
\hline $\mathrm{C}$ & 3.341068 & 0.389324 & -1.632600 \\
\hline $\mathrm{C}$ & 3.670160 & -0.612915 & -2.563637 \\
\hline $\mathrm{C}$ & 4.211163 & -0.280067 & -3.810134 \\
\hline $\mathrm{C}$ & 4.434063 & 1.057376 & -4.148391 \\
\hline $\mathrm{C}$ & 4.114495 & 2.062651 & -3.230393 \\
\hline $\mathrm{C}$ & 3.571331 & 1.732998 & -1.985450 \\
\hline $\mathrm{C}$ & 3.504365 & 1.115530 & 1.115729 \\
\hline $\mathrm{C}$ & 4.883053 & 0.955973 & 1.352535 \\
\hline $\mathrm{C}$ & 5.569560 & 1.835689 & 2.192748 \\
\hline $\mathrm{C}$ & 4.900501 & 2.913410 & 2.786078 \\
\hline $\mathrm{C}$ & 3.536948 & 3.095950 & 2.541580 \\
\hline $\mathrm{C}$ & 2.843920 & 2.194864 & 1.724974 \\
\hline $\mathrm{C}$ & 3.285094 & -1.668043 & 0.36859 \\
\hline
\end{tabular}




\begin{tabular}{|c|c|c|c|}
\hline $\mathrm{P}$ & -2.437420 & 0.860660 & 5.295516 \\
\hline $\mathrm{P}$ & 2.192941 & -1.501938 & 5.150272 \\
\hline $\mathrm{P}$ & -2.591845 & 0.166763 & -0.122763 \\
\hline $\mathrm{P}$ & 2.572009 & -0.014350 & -0.006185 \\
\hline Mo & -0.158053 & -0.357516 & 5.082566 \\
\hline Mo & -0.004643 & 0.010516 & -0.009775 \\
\hline $\mathrm{N}$ & -0.061134 & -0.072516 & 3.139282 \\
\hline $\mathrm{N}$ & -0.048158 & 0.015779 & 1.955914 \\
\hline $\mathrm{N}$ & 0.823512 & 1.263979 & 6.191031 \\
\hline $\mathrm{N}$ & -0.358475 & -0.826828 & 7.170286 \\
\hline $\mathrm{N}$ & -1.281970 & -2.208318 & 5.200640 \\
\hline $\mathrm{N}$ & 0.081116 & -2.119276 & -0.445934 \\
\hline $\mathrm{N}$ & 0.042573 & -0.187774 & -2.152755 \\
\hline $\mathrm{N}$ & -0.007809 & 2.051492 & -0.839007 \\
\hline $\mathrm{H}$ & 1.452630 & 2.326695 & 4.520614 \\
\hline $\mathrm{H}$ & 2.580095 & 4.132214 & 5.793000 \\
\hline $\mathrm{H}$ & 2.580128 & 4.021829 & 8.330509 \\
\hline $\mathrm{H}$ & 1.454911 & 2.084920 & 9.423481 \\
\hline $\mathrm{H}$ & 0.470592 & 0.386558 & 10.222046 \\
\hline $\mathrm{H}$ & -1.695977 & -3.188291 & 9.201868 \\
\hline $\mathrm{H}$ & -2.570307 & -4.258650 & 7.585827 \\
\hline $\mathrm{H}$ & -3.479625 & -5.417665 & 5.579221 \\
\hline $\mathrm{H}$ & -2.959893 & -4.441841 & 3.293275 \\
\hline $\mathrm{H}$ & -1.553537 & -2.403955 & 3.150404 \\
\hline $\mathrm{H}$ & -4.937278 & 2.395112 & 4.696819 \\
\hline $\mathrm{H}$ & -5.354053 & 4.509834 & 3.494487 \\
\hline $\mathrm{H}$ & -3.454011 & 5.811063 & 2.543851 \\
\hline $\mathrm{H}$ & -1.122557 & 4.967547 & 2.803621 \\
\hline $\mathrm{H}$ & -0.705975 & 2.781986 & 3.927992 \\
\hline $\mathrm{H}$ & -1.574029 & 3.140851 & 6.918926 \\
\hline $\mathrm{H}$ & -2.137034 & 3.935830 & 9.190398 \\
\hline $\mathrm{H}$ & -3.788282 & 2.687227 & 10.588125 \\
\hline $\mathrm{H}$ & -4.820885 & 0.596579 & 9.703077 \\
\hline $\mathrm{H}$ & -4.256829 & -0.216615 & 7.449524 \\
\hline $\mathrm{H}$ & -3.821697 & -0.458483 & 3.785983 \\
\hline $\mathrm{H}$ & -3.825482 & -1.139581 & 5.421031 \\
\hline $\mathrm{H}$ & -4.833476 & 0.285881 & 5.063452 \\
\hline $\mathrm{H}$ & 4.019954 & -0.095004 & 7.085808 \\
\hline $\mathrm{H}$ & 4.818323 & -0.670301 & 9.341094 \\
\hline $\mathrm{H}$ & 4.070075 & -2.780072 & 10.447549 \\
\hline $\mathrm{H}$ & 2.470578 & -4.291306 & 9.264966 \\
\hline $\mathrm{H}$ & 1.687690 & -3.741687 & 6.992578 \\
\hline $\mathrm{H}$ & 0.584996 & -3.870731 & 4.485127 \\
\hline $\mathrm{H}$ & 1.152332 & -6.137312 & 3.657621 \\
\hline $\mathrm{H}$ & 5.268920 & -4.942037 & 3.192186 \\
\hline $\mathrm{H}$ & 4.728428 & -2.705634 & 4.074850 \\
\hline
\end{tabular}




$\begin{array}{lrrr}\mathrm{H} & 3.367101 & 0.619928 & 4.947219 \\ \mathrm{H} & 4.511900 & -0.732523 & 4.766525 \\ \mathrm{H} & 3.399854 & -0.288602 & 3.432907 \\ \mathrm{H} & 0.186861 & -2.733455 & 1.537405 \\ \mathrm{H} & 0.358460 & -5.149214 & 1.031427 \\ \mathrm{H} & 0.323422 & -5.906432 & -1.393521 \\ \mathrm{H} & 0.177634 & -4.158834 & -3.163638 \\ \mathrm{H} & 0.086991 & -2.572627 & -4.560677 \\ \mathrm{H} & 0.111345 & 1.709685 & -4.957438 \\ \mathrm{H} & 0.070502 & 3.513041 & -3.902898 \\ \mathrm{H} & -0.049960 & 5.580425 & -2.518958 \\ \mathrm{H} & -0.190133 & 5.327677 & 0.000529 \\ \mathrm{H} & -0.165291 & 3.042478 & 0.976594 \\ \mathrm{H} & -2.370991 & -2.751779 & -0.314524 \\ \mathrm{H} & -3.803785 & -4.629363 & 0.371423 \\ \mathrm{H} & -5.925244 & -4.195759 & 1.639381 \\ \mathrm{H} & -6.586208 & -1.847909 & 2.155362 \\ \mathrm{H} & -5.189978 & 0.035226 & 1.417073 \\ \mathrm{H} & -3.304157 & -1.664584 & -2.349932 \\ \mathrm{H} & -4.184774 & -1.301474 & -4.622396 \\ \mathrm{H} & -4.715357 & 1.005228 & -5.418729 \\ \mathrm{H} & -4.354883 & 2.945347 & -3.898117 \\ \mathrm{H} & -3.461348 & 2.604391 & -1.630726 \\ \mathrm{H} & -2.654909 & 2.518658 & 0.447954 \\ \mathrm{H} & -4.283977 & 1.816637 & 0.608894 \\ \mathrm{H} & -3.025113 & 1.511767 & 1.853611 \\ \mathrm{H} & 3.522968 & -1.667512 & -2.320437 \\ \mathrm{H} & 4.476522 & -1.073087 & -4.513505 \\ \mathrm{H} & 4.870727 & 1.316634 & -5.116004 \\ \mathrm{H} & 4.306677 & 3.109564 & -3.481328 \\ \mathrm{H} & 3.347151 & 2.531750 & -1.274307 \\ \mathrm{H} & 5.436896 & 0.143267 & 0.877988 \\ \mathrm{H} & 6.635569 & 1.682560 & 2.381971 \\ \mathrm{H} & 5.446396 & 3.611542 & 3.426226 \\ \mathrm{H} & 1.77605834 & 3.943013 & 2.984836 \\ \mathrm{H} & 2.833666 & -2.419010 & -0.292819 \\ \mathrm{H} & -0.332453 & 1.556110 \\ \mathrm{H} & -1.702114 & 0.238352 \\ & -1.687120 & 10.937535 \\ \mathrm{H} & -0.550524 & -6.017794\end{array}$


V. DFT-Computed Qualitative Molecular Orbital Diagram for $\left[\left({ }^{\mathrm{Ph}} \mathrm{Tpy}\right)\left(\mathrm{PPh}_{2} \mathbf{M e}\right)_{2} \mathbf{M o}(\mathrm{Cl})\right]$
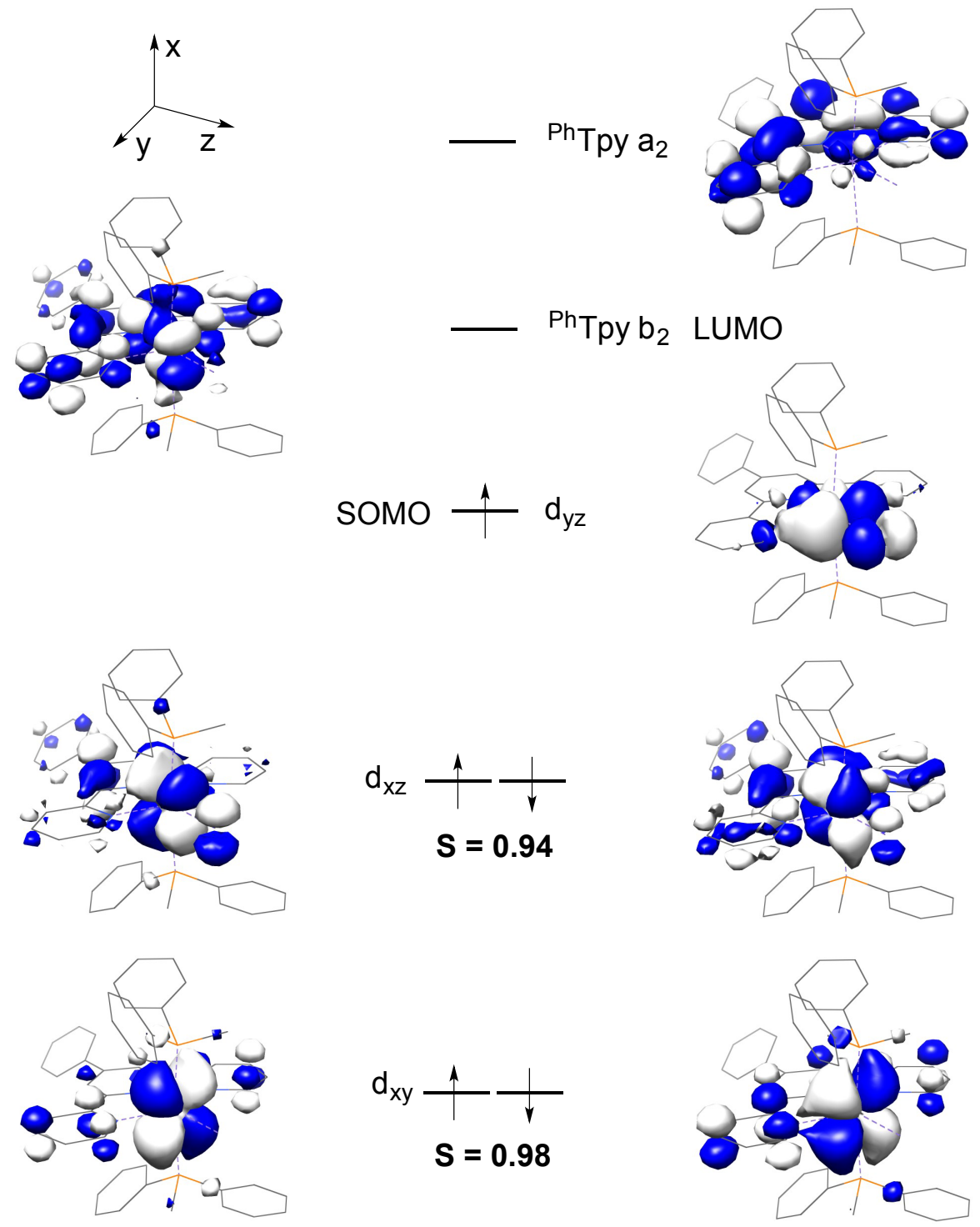

Figure S12. Qualitative molecular orbital diagram for $\left[\left({ }^{\mathrm{Ph}} \mathrm{Tpy}\right)\left(\mathrm{PPh}_{2} \mathrm{Me}\right)_{2} \mathrm{Mo}(\mathrm{Cl})\right]$ from a spin-unrestricted B3LYP DFT calculation. 


\section{Additional Mulliken Spin Density Plots}

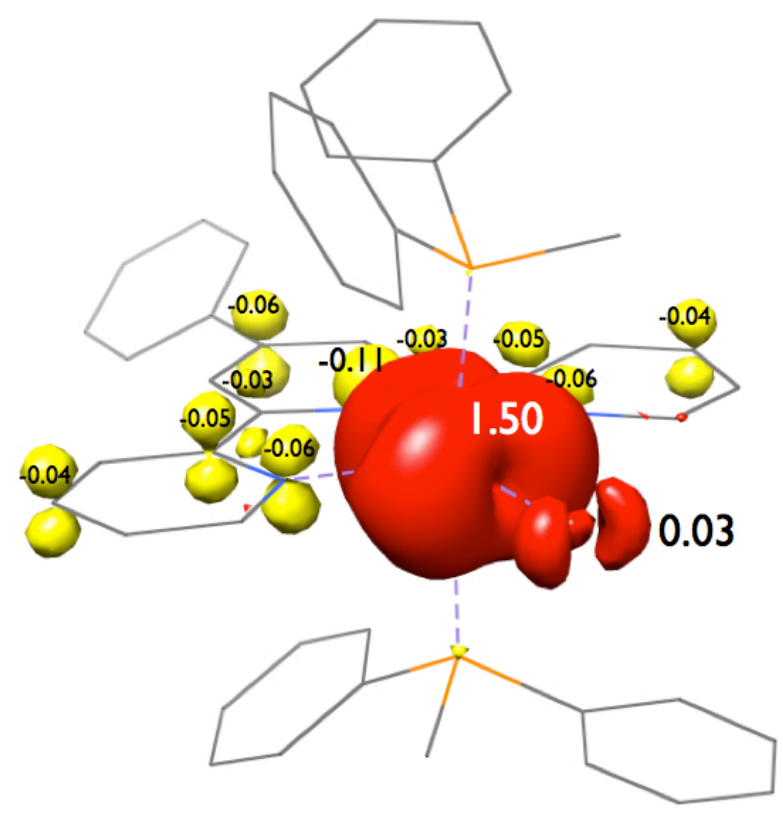

Figure S13. Mulliken spin density plot for $\left[\left({ }^{\mathrm{Ph}} \mathrm{Tpy}\right)\left(\mathrm{PPh}_{2} \mathrm{Me}\right)_{2} \mathrm{Mo}(\mathrm{Cl})\right]$ from a spinunrestricted B3LYP DFT calculation.

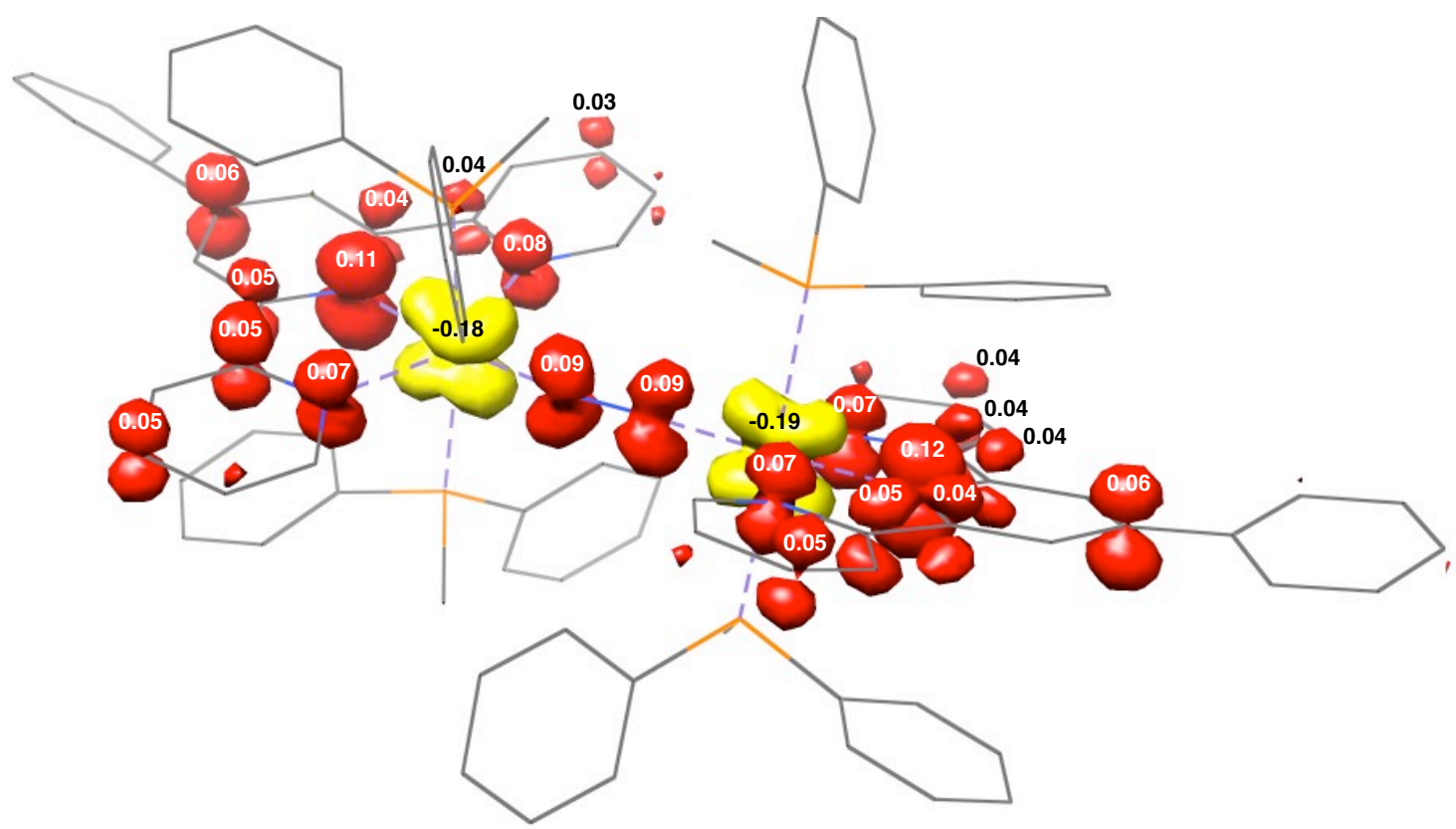

Figure S14. Mulliken spin density plot for $\left[\left\{\left({ }^{\mathrm{Ph}} \mathrm{Tpy}\right)\left(\mathrm{PPh}_{2} \mathrm{Me}\right)_{2} \mathrm{Mo}_{2}\left(\mu_{2}-\mathrm{N}_{2}\right)\right]\left[\mathrm{BArF}{ }^{24}\right]\right.$ from a spin-unrestricted B3LYP DFT calculation. 


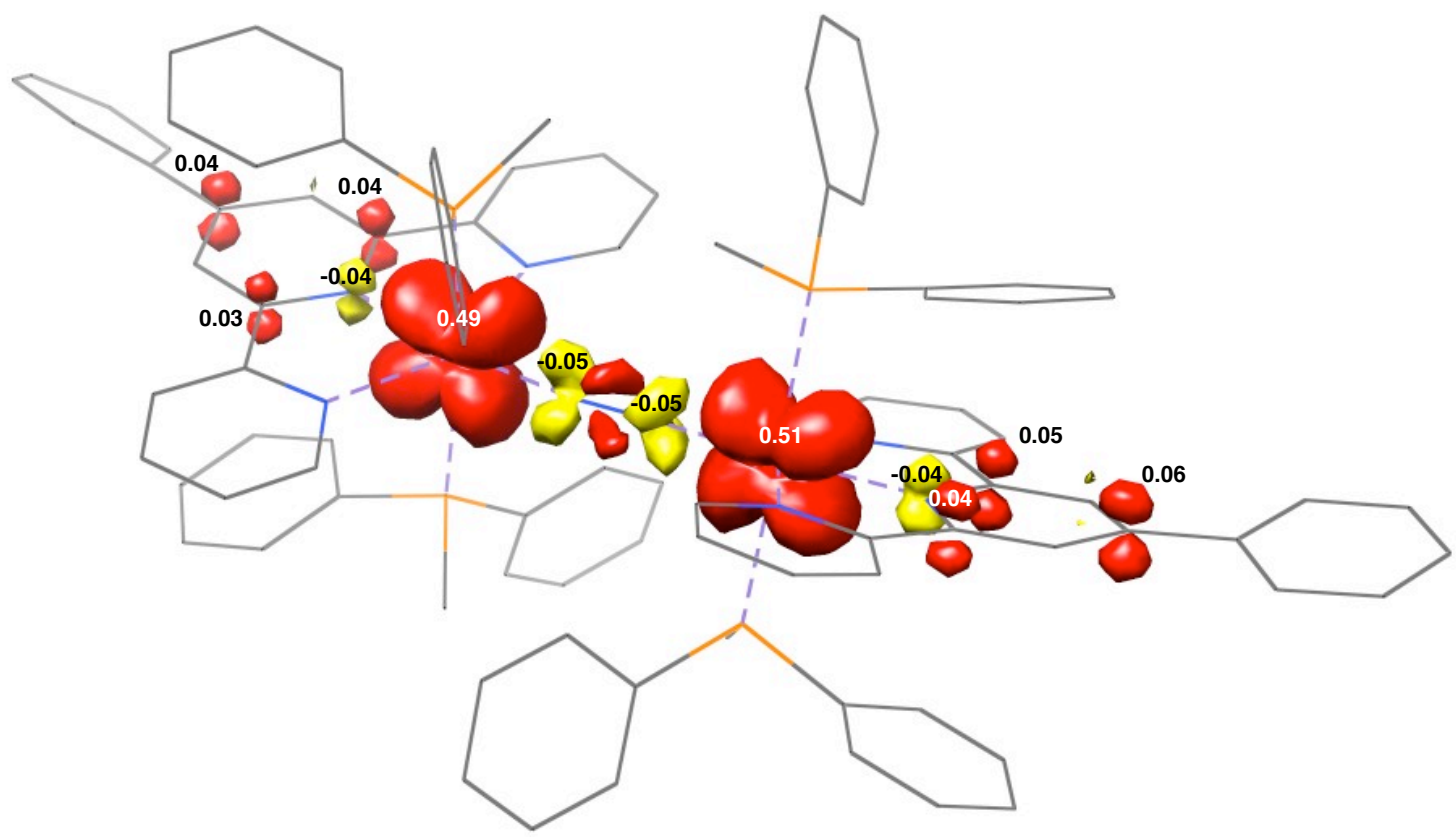

Figure S15. Mulliken spin density plot for $\left[\left\{\left({ }^{\mathrm{Ph}} \mathrm{Tpy}\right)\left(\mathrm{PPh}_{2} \mathrm{Me}\right)_{2} \mathrm{Mo}\right\}_{2}\left(\mu_{2}-\mathrm{N}_{2}\right)\right]\left[\mathrm{BArF}{ }^{24}\right]_{3}$ from a spin-unrestricted B3LYP DFT calculation. 